\title{
The influence of plasma glucose upon pulsatile ocular blood flow in subjects with Type II diabetes mellitus
}

\author{
R.L. Perrott ${ }^{1}$, R. V. North ${ }^{1}$, N. Drasdo ${ }^{1}$, K. A. Ahmed ${ }^{2}$, D. R. Owens ${ }^{2}$ \\ ${ }^{1}$ Department of Optometry and Vision Sciences, Cardiff University, Cardiff, UK \\ ${ }^{2}$ Diabetes Research Unit, UWCM, Cardiff, UK
}

\section{Abstract}

Aims/hypothesis. This study investigated the influence of plasma glucose upon pulsatile ocular blood flow in subjects with Type II (non-insulin-dependent) diabetes mellitus.

Methods. A total of 19 subjects with Type II diabetes and 8 normal control subjects undertook a meal tolerance test after an overnight fast. The pulsatile ocular blood flow, using the Ocular Blood Flow Tonometer, and plasma glucose concentrations were taken at times $0 \mathrm{~min}, 90 \mathrm{~min}$ and $240 \mathrm{~min}$. Blood pressure and glycated haemoglobin concentrations, in the subjects with diabetes, were also measured at time $0 \mathrm{~min}$. Pulsatile ocular blood flow and plasma glucose were also measured at times 0 and $90 \mathrm{~min}$ in 5 subjects with Type II diabetes mellitus who remained fasting.

Results. It was found that the subjects with diabetes who undertook the meal tolerance test showed a significant increase in both plasma glucose concentrations and pulsatile ocular blood flow from time 0-90 $\mathrm{min}$, followed by a decrease from $90 \mathrm{~min}$ to the end of the session at $240 \mathrm{~min} . \quad(p<0.001$ in each case). Regression analysis showed a significant correlation between the change in pulsatile ocular blood flow and the change in plasma glucose concentration $(r=0.671, p=0.001)$. Control subjects showed no significant change in either plasma glucose or pulsatile ocular blood flow during the meal tolerance test. Subjects with diabetes mellitus who remained fasting also showed no significant change in pulsatile ocular blood flow or plasma glucose concentrations. No correlation was found between glycated haemoglobin concentrations or blood pressure and pulsatile ocular blood flow.

Conclusion/interpretation. Pulsatile ocular blood flow is influenced by changes in plasma glucose concentrations in Type II diabetes mellitus, indicating that uncontrolled hyperglycaemia might result in a higher pulsatile ocular blood flow than might otherwise be expected. [Diabetologia (2001) 44: 700-705]

Keywords Plasma glucose, pulsatile ocular blood flow, Type II diabetes mellitus, meal tolerance test, hyperglycaemia, fasting
The development of diabetic retinopathy is a major concern in the management of diabetes mellitus. Pre-

Received: 7 November 2000 and in revised form: 6 February 2001

Corresponding author: R.V. North PhD, Department of Optometry and Vision Sciences, Cardiff University, Redwood Building, King Edward VII Avenue, Cardiff, CF1 3XF, UK Abbreviations: POBF, Pulsatile ocular blood flow; IOP, Intraocular pressure; $\mathrm{HbA1}_{c}$, glycated haemoglobin; MTT, meal tolerance test; EDRF, endothelium derived relaxation factor. vious research into this complication, using techniques such as fluorescein angiography [1] and Laser Doppler velocimetry [2-5], has contributed to the understanding of the process and progression of retinopathy $[2,4-7]$.

Clinical studies have shown that ocular haemodynamic changes in diabetes are complex and still have to be fully defined. Generally an initial reduction in retinal blood flow velocity has been observed in the early stages of diabetes before the onset of any clinically recognisable retinopathy $[4,5,7,9,10]$. A positive transition is then seen as retinal blood speed in- 
creases as retinopathy progresses [6-8]. This correlates with an early reduction in volumetric flow, which then increases with the evolution of retinopathy $[1,8-10]$. A progressive increase in vessel diameter has also been unequivocally observed $[5,8,10]$. Some contradictory results have been reported where severity of retinopathy has been correlated with a progressive reduction in blood flow with no subsequent increase [11] but the aforementioned results are the most widely accepted.

The plasma glucose concentration has also proved to be a contributory factor, to changes in retinal blood flow with deviations in plasma glucose concentrations, in either direction, associated with a corresponding variation in retinal blood flow [1, 7]. These alterations to the retinal haemodynamics have been attributed to a breakdown in the autoregulation of the retinal vasculature. Moreover, consistency of metabolic control has also been closely associated with the degree of diabetic retinopathy. An increase of glycated haemoglobin concentrations $\left(\mathrm{HbA} 1_{\mathrm{c}}\right)$, indicating poor long term metabolic control, is concurrent with a subsequent increase in the risk of development of microangiopathy, of which retinopathy is a major constituent [12-15]. Therefore, according to current knowledge, hyperglycaemia is associated with the development of diabetic retinopathy and with a change in retinal haemodynamics.

Studies of ocular haemodynamics have focused on retinal blood flow, but only $5 \%$ of the total ocular blood flow can be attributed to this parameter [16]. Interest in a more comprehensive assessment of the haemodynamics of the entire ocular circulation has been prompted by the development of a clinically feasible technique to measure pulsatile ocular blood flow (POBF). This can be quickly and non-invasively measured using the Ocular Blood Flow (OBF) tonometer (OBF Labs UK). The theory of POBF has been well documented in previous studies [17-19]. It is based on pneumatonographic measurement of the variation of eye volume, caused by a pulsatile bolus of blood entering the eye during systole. This causes a resultant variation in intra-ocular pressure (IOP), known as the pulse amplitude (PA). Five pulse cycles are recorded, which takes approximately 10 to $15 \mathrm{~s}$. The OBF software then averages these values and calculates the volume of blood passing through the eye in a given time, either per minute or per second. Measures of pulse rate, IOP and PA are also provided.

The OBF tonometer has been developed primarily to assess ocular blood flow in glaucoma. However, recent studies have assessed POBF within the diabetic population. Preliminary results appear to suggest concordance with changes found in the retinal blood flow in the presence of diabetic retinopathy, although POBF appears to be a much less sensitive indicator as a more advanced form of retinopathy needs to be apparent before changes are seen [20-22].

The influence of plasma glucose concentrations upon POBF measurements is controversial. Investigators [23] have shown that alterations in plasma glucose concentrations had no effect on choroidal blood flow by using the Heidelberg Retina Flowmeter. Also no correlation between POBF and $\mathrm{HbA} 1_{c}$ was found.

The OBF tonometer is a simple, non-invasive device that can be used clinically to assess a much larger portion of ocular blood flow than previous techniques that have concentrated measuring only retinal flow. Retinal blood flow is known to be affected by plasma glucose concentrations but the effect on POBF remains controversial. Therefore this study aims to investigate the influence of plasma glucose concentrations upon POBF in patients with Type II diabetes mellitus.

\section{Subjects and methods}

A total of 19 subjects with Type II diabetes mellitus were recruited from the Diabetes Research Unit, Llandough Hospital. The subjects were aged between 51 and 78 years, (mean and SD 60.2 years \pm 6.4 ) including 13 men and 6 women. Refractive errors were between +4.00 and -2.00 dioptres spectacle refraction with a maximum cyl of 1.50 dioptres. Intraocular pressure (IOP) was less than $21 \mathrm{mmHg}$ in all subjects.

The majority of subjects had a known duration of diabetes of 2 years or less. The mean (SD) being 1.2 years $( \pm 2.3)$. All medication was noted and any subjects with peripheral vascular disease including poorly controlled hypertension were excluded. All subjects were controlled by either diet alone or by oral hypoglycaemic agents. No subjects were receiving insulin. None exhibited any ophthalmoscopically visible diabetic retinopathy, with the exception of one subject who had very mild background retinopathy consisting of less than four microaneurysms. None of the subjects had any other current or previous ocular disorders or family history of glaucoma. Fundus examination was carried out by two $45^{\circ}$ fundus photographs taken using a Canon CR4 NM 45, with pupils dilated using $1 \%$ tropicamide. Photographs were then assessed by trained retinopathy graders.

A group of 8 control subjects matched for age were recruited with an age range of 62-69 years (66.3 years \pm 2.2 , mean and SD). All control subjects were within the above range of refractive error ( +4.00 and -2.00 dioptres) with the exception of one subject who had a mean spherical refractive error of -5.00 dioptres. These subjects had no history of ocular disorders or family history of glaucoma and IOP was less than $21 \mathrm{mmHg}$.

The study was approved by the local ethics committee and carried out after informed consent from all subjects.

Ocular Blood Flow Measurements. Pulsatile Ocular Blood Flow (POBF) was measured when plasma glucose concentrations were firstly, changing during a standardised meal tolerance test and secondly, relatively stable during a period of fasting. The POBF was measured using the Langham OBF Tonometer (OBF Labs UK) [19-20]. Measurements were taken from the right eye, except in circumstances where this was 
Table 1. Summary of Mean ( \pm SD) values of BP, plasma glucose and POBF measurements for control subjects and Type II diabetes subjects

\begin{tabular}{|c|c|c|c|}
\hline$n$ & $\begin{array}{l}\text { Control subjects during } \\
\text { MTT (mean and SD) } \\
8\end{array}$ & $\begin{array}{l}\text { Type II diabetes during } \\
\text { MTT (mean and SD) } \\
19\end{array}$ & $\begin{array}{l}\text { Type II diabetes fast- } \\
\text { ing (mean and SD) } \\
5\end{array}$ \\
\hline Systolic blood pressure (mmHg) & $\mathbf{1 3 6 . 2 7} \pm 0.16$ & $\mathbf{1 4 2 . 5 8} \pm 25.15$ & $\mathbf{1 5 2 . 4} \pm 19.19$ \\
\hline Pulse pressure $(\mathrm{mmHg})$ & $\mathbf{5 6 . 7 1} \pm 10.9$ & $\mathbf{6 1 . 0 5} \pm 20.41$ & $\mathbf{6 9 . 6} \pm 13.96$ \\
\hline Mean blood pressure $(\mathrm{mmHg})$ & $\mathbf{7 5 . 6 2} \pm 14.62$ & $\mathbf{1 0 1 . 8 8} \pm 14.02$ & $\mathbf{1 1 0 . 4} \pm 11.52$ \\
\hline Mean duration of diabetes (years) & NA & $\mathbf{1 . 3 6} \pm 2.37$ & $\mathbf{0 . 6 1 6} \pm 0.88$ \\
\hline $90 \mathrm{~min}$ & $\mathbf{5 . 3 2} \pm 0.76$ & $\mathbf{1 2 . 0 6} \pm 4.44$ & $\mathbf{6 . 6 9} \pm 0.91$ \\
\hline $240 \mathrm{~min}$ & $\mathbf{4 . 9 9} \pm 0.42$ & $7.47 \pm 3.97$ & NA \\
\hline $\operatorname{POBF}\left(\mu \mathrm{lsec}{ }^{-1}\right)$ at $: 0 \mathrm{~min}$ & $\mathbf{1 9 . 7 2} \pm 8.01$ & $\mathbf{1 4 . 5 5} \pm 5.87$ & $\mathbf{1 4 . 5 4} \pm 2.49$ \\
\hline $90 \mathrm{~min}$ & $\mathbf{2 0 . 6 8} \pm 7.45$ & $\mathbf{1 7 . 2 0} \pm 6.79$ & $\mathbf{1 5 . 3 8} \pm 2.08$ \\
\hline $240 \mathrm{~min}$ & $\mathbf{1 8 . 4 7} \pm 6.97$ & $\mathbf{1 4 . 3 6} \pm 5.89$ & $\mathbf{N A}$ \\
\hline IOP $(\mathrm{mmHg})$ & $\mathbf{1 5 . 8 4} \pm 3.66$ & $\mathbf{1 4 . 5 1} \pm 4.25$ & $\mathbf{1 4 . 8 8} \pm 1.37$ \\
\hline Heart rate (beats per minute) & $\mathbf{6 4 . 8 5} \pm 6.64$ & $\mathbf{6 4} \pm 7.2$ & $\mathbf{6 3 . 9 7} \pm 8.2$ \\
\hline
\end{tabular}

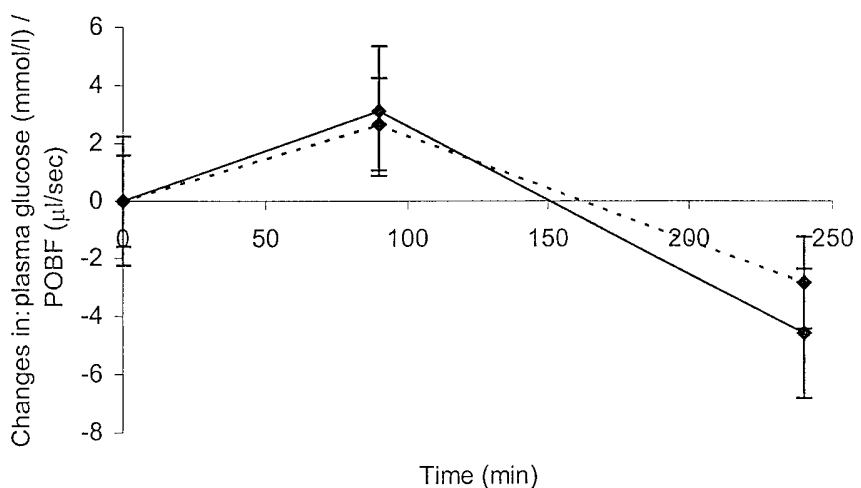

Fig. 1. Mean $( \pm \mathrm{SD})$ change in plasma glucose concentration and POBF for subjects with Type II diabetes during the MTT (measured from the baseline initial value. Bars represent \pm 1SD) Key: - - - POBF, — Plasma Glucose

precluded, that is when the eye fell outside the aforementioned criteria, or for reasons, including patient apprehension, a reading could not be gained within the recommended three attempts. A single trained optometrist carried out all measurements (RP).

The cornea was anaesthetised using one drop combination local anaesthetic and stain - proxymetacaine $(0.5 \%)$ and fluorescein sodium $(0.25 \%)$. This provided adequate anaesthesia with the minimum amount of discomfort and the minimum risk of corneal desquamation [24]. It also provided the ready presence of a stain to assess corneal integrity after the measurement was taken. All measurements were taken with the patient in a sitting position and recorded in $\mu \mathrm{l}$ per second.

Meal Tolerance test. Plasma glucose concentrations increased during a standardised meal tolerance test (MTT) which was consumed over a $10 \mathrm{~min}$ period providing a total of 482.6 Kcal, (20\% protein, $23 \%$ fat and $58 \%$ carbohydrates). All subjects fasted overnight for $10 \mathrm{~h}$ and diabetic subjects ceased any oral hypoglycaemic agents for $24 \mathrm{~h}$ before testing.

Before and after the administration of the food, mixed venous blood samples were taken at regular intervals for $4 \mathrm{~h}$. Plasma glucose concentrations were then measured from the blood samples using an automated colorimetric method [25]. $\mathrm{HbA}_{1 \mathrm{c}}$ was measured at time 0 min for each subject with diabetes mellitus by an ion exchange method (test combination $\mathrm{HbA}_{1 \mathrm{c}}$ BCL, Lewes, Sussex, UK).

POBF and plasma glucose were measured at time $0 \mathrm{~min}$, i.e. at fasting plasma glucose concentrations, before the administration of the meal and at 90 and 240 minutes from the commencement of the meal. These times were chosen as they approximately coincided with the expected peak glucose concentrations ( $90 \mathrm{~min}$ ) and at the end of the test [26]. Corneal integrity was checked under both white light and cobalt blue light both prior to, and after measurements were taken. The POBF measurements were recorded immediately after the blood samples were taken.

Blood pressure (BP) was measured at 0 min in all subjects. The study was carried out blind, in that different persons determined the plasma glucose concentrations and measurements of POBF.

Fasting. OBF was recorded in 5 of the subjects with Type II diabetes mellitus during a prolonged fast. In these cases the subjects fasted overnight and plasma glucose and POBF were measured at time $0 \mathrm{~min}$ and $90 \mathrm{~min}$. Blood pressure and $\mathrm{HbA}_{1 \mathrm{c}}$ were also measured at time $0 \mathrm{~min}$.

\section{Results}

A summary of the results are shown in Table 1.

Plasma glucose concentrations in the subjects with Type II diabetes mellitus that undertook the MTT followed the expected pattern. The mean (SD) starting value was $8.9 \mathrm{mmol} / \mathrm{l}( \pm 3.5)$ which increased after $90 \mathrm{~min}$ to $12.1 \mathrm{mmol} / \mathrm{l}( \pm 4.4)$ and was then found to have decreased after $240 \mathrm{~min}$ to $7.5 \mathrm{mmol} / \mathrm{l}( \pm 4)$. POBF was also found to follow a similar trend. The mean (SD) initial value was $14.5 \mu \mathrm{l} / \mathrm{sec}( \pm 5.9)$. This increased to $17.2 \mu \mathrm{l} / \mathrm{sec}( \pm 6.8)$ after $90 \mathrm{~min}$, then decreased to $14.4 \mu \mathrm{l} / \mathrm{sec}( \pm 5.9)$ at $240 \mathrm{~min}$. The change in POBF values and plasma glucose concentrations between each test time are shown in Fig.1. Plasma 


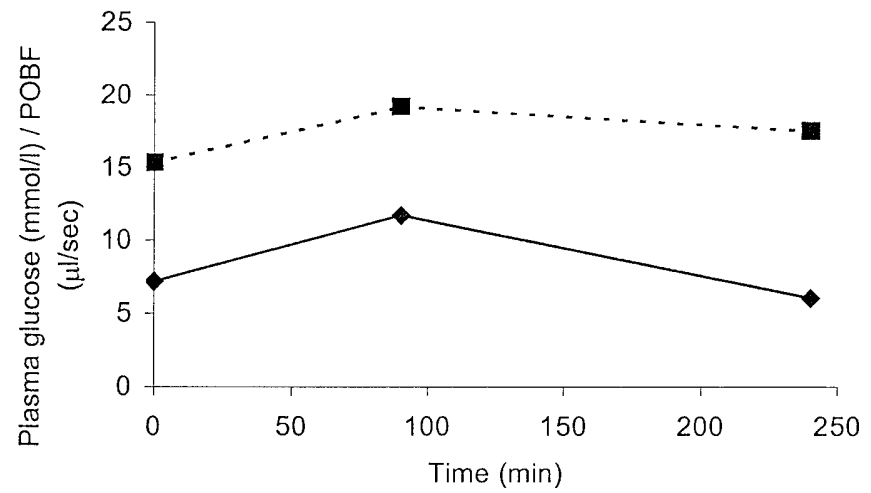

Fig. 2. Absolute values for a single subject (ML) with diabetes. Key: - - - POBF, — Plasma Glucose

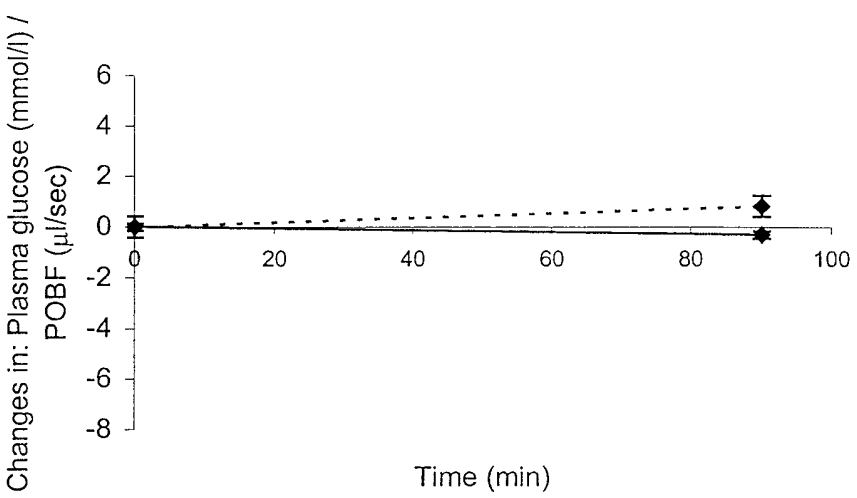

Fig. 3. Mean $( \pm \mathrm{SD})$ change of plasma glucose concentration and POBF for subjects with Type II diabetes during fasting, (measured from the baseline initial value. Bars represent \pm 1SD). Key: - - - - POBF, — Plasma Glucose

glucose showed a mean (SD) increase of $+3.1 \mathrm{mmol} / 1$ $( \pm 1.7)$ after $90 \mathrm{~min}$ which decreased by about $4.60 \mathrm{mmol} / \mathrm{l}( \pm 1.8)$ from 90 to $240 \mathrm{~min}$. Furthermore, POBF showed mean changes of $+2.6 \mu \mathrm{l} / \mathrm{sec}( \pm 2.5)$ and $-2.84 \mu \mathrm{l} / \mathrm{sec}( \pm 3.04)$ after 90 and $240 \mathrm{~min}$ respectively. Wilcoxon statistical analysis showed all changes to be statistically significant $(p<0.001)$. Figure 2 shows the POBF and plasma glucose concentrations for one subject (ML). Regression analysis showed a significant positive correlation between the change in POBF values and change in plasma glucose concentrations, $(\mathrm{r}=0.67, p=0.001)$.

Subjects with Type II diabetes who were fasting showed no significant change of plasma glucose concentrations or POBF (Fig. 3). Mean (SD) values for plasma glucose were $6.98 \mathrm{mmol} / \mathrm{l}( \pm 1.1)$ and $6.69 \mathrm{mmol} / \mathrm{l}( \pm 0.9)$, at 0 and $90 \mathrm{~min}$ respectively. The mean (SD) POBF values were, $14.54 \mu \mathrm{l} / \mathrm{sec}( \pm 2.5)$ and $15.38 \mu \mathrm{l} / \mathrm{sec}( \pm 2.1)$ at these two times. This corresponded to mean changes over the $90 \mathrm{~min}$ of about $0.29 \mathrm{mmol} / \mathrm{l} \quad( \pm 0.4)$, and $+0.8 \mu \mathrm{l} / \mathrm{sec} \quad( \pm 2)$, which were not significant ( $p=0.59$ in each case).

Figure 4 shows there was no significant change in plasma glucose concentrations or POBF during the

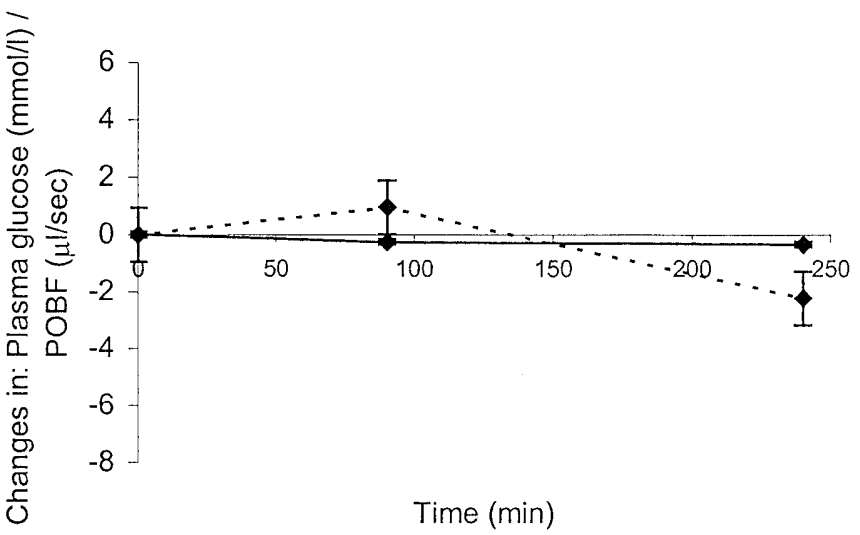

Fig. 4. Mean $( \pm \mathrm{SD})$ change of plasma glucose concentration and POBF for control subjects during the MTT, (measured from the baseline initial value. Bars represent \pm 1SD). Key: - . - POBF, — Plasma Glucose

MTT for the control subjects. Mean fasting plasma glucose concentration was $5.6 \mathrm{mmol} / \mathrm{l}( \pm 0.3)$ at time $0 \mathrm{~min}, \quad 5.3 \mathrm{mmol} / \mathrm{l}( \pm 0.8)$ at time $90 \mathrm{~min}$, and $5.0 \mathrm{mmol} / \mathrm{l}( \pm 0.4)$ at time $240 \mathrm{~min}$. Mean POBF values were $19.7 \mu \mathrm{l} / \mathrm{sec}( \pm 8.0)$ at time $0 \mathrm{~min}, 20.68 \mu \mathrm{l} /$ $\sec ( \pm 7.5)$ at time $90 \mathrm{~min}$ and $18.5 \mu \mathrm{l} / \mathrm{sec}( \pm 7.0)$ at time $240 \mathrm{~min}$. Changes in plasma glucose concentrations showed a decrease of about $0.2 \mathrm{mmol} / \mathrm{l}( \pm 0.7)$ between 0 and $90 \mathrm{~min} \quad(p=0.46)$, and about $0.3 \mathrm{mmol} / \mathrm{l} \quad( \pm 0.7)$ between 90 and $240 \mathrm{~min}$ $(p=0.40)$. Changes in POBF were, $+1.0 \mu \mathrm{l} / \mathrm{sec}$ $( \pm 2.4) \quad(p=0.27)$ from $0-90 \mathrm{~min}$ and $-2.2 \mu \mathrm{l} / \mathrm{sec}$ ( \pm 3.6) from $90-240 \mathrm{~min}(p=0.55)$.

Mean BP was found to be significantly higher in the subjects with Type II diabetes compared with control subjects $(p=0.002)$. No statistical difference was found between pulse pressure or PA between the groups, $(p=0.56$ and $p=0.45$ respectively $)$ and PA showed very little variation in either group over the test time. Baseline POBF was lower in the diabetic group compared to normal subjects but this difference was not significant $(p=0.08)$. Mann-Whitney statistical analysis was used in these cases.

No relationship was found between POBF or changes in POBF during the meal test with either systolic or diastolic blood pressure, mean blood pressure or pulse pressure in all groups. There was no correlation between the fasting plasma glucose concentration or $\mathrm{HbA}_{1 \mathrm{c}}$ with POBF as measured at time 0 in subjects with Type II diabetes. Only the change in plasma glucose during the meal test was correlated with the change in POBF in subjects with Type II diabetes.

\section{Discussion}

Our results show that pulsatile ocular blood flow is altered by changes in plasma glucose concentrations in 
subjects with Type II diabetes mellitus. In contrast control subjects consuming a standardised test meal showed no significant variation in either plasma glucose concentration or POBF values after 90 and 240 min time points. When the plasma glucose concentration was relatively constant during a prolonged fast in subjects with diabetes, no significant variation of POBF was found. Subjects with diabetes who consumed the test meal showed an increased plasma glucose 90 minutes after the meal which then returned to baseline concentrations after $240 \mathrm{~min}$. POBF was observed to follow the same course. Our results have shown that a higher plasma glucose concentration can be associated with an increased pulsatile ocular blood flow in subjects with diabetes mellitus. These findings are in agreement with current studies on retinal blood flow changes in the presence of hyperglycaemia [1,7]. The use of fluorescein angiography and glucose clamp methods has shown that acute elevations in plasma glucose concentrations caused an increase in retinal blood flow [1]. Conversely, by the use of a bi-directional laser velocimetry, a reduction in plasma glucose has been associated with a fall in retinal blood flow as the subjects were changed from hyperglycaemia to normoglycaemia [7].

We also found a lower POBF in subjects with diabetes compared with control subjects. This finding contradicts a previous study [20] which found that diabetic subjects had a higher POBF. However, the subjects of this study had a longer duration of diabetes mellitus and a wider range of retinopathy from no retinopathy to pre-proliferative retinopathy. This variation could account for the differences seen. Our observation of a reduced POBF agrees with previous studies relating to retinal flow, where a reduction was seen in the early stages of diabetes before the onset of visible retinopathy $[1,3,5,8,10]$. Currently no explanation for this lower POBF in subjects with Type II diabetes has been found but could be due to rheological changes associated with diabetes.

This study demonstrated that higher plasma glucose concentrations are associated with an increase in the pulsatile portion of ocular blood flow. Heightened blood flow concentrations, whether they are chronic or acute, can increase the shear stress concentrations exerted on the vascular cell wall. This mechanical action of shear stress could be a contributory factor in the selective loss of pericytes, which is a primary feature of diabetic vascular deterioration [27, 28]. This loss could be causative of the hypoxic situation in the diabetic retina and the evolution of diabetic retinopathy.

The physiology of the increased flow seen in this study has yet to be determined. While the presence of increased glucose concentrations could augment the activity of nitric oxide synthase and hence nitric oxide release [29] this is not likely to account for the increased flow seen in this study. However, excessive glucose concentrations have been shown to result in an overproduction of lactate [28]. This could be the effector in pre-capillary vasodilation [30] and subsequent increases in blood flow seen in acute hyperglycaemia in this study on pulsatile flow, and previous work focused on retinal flow $[1,7,31]$. However, this hypothesis remains to be proved.

The variable and contradictory results seen in blood flow studies regarding the pulsatile or choroidal portions of ocular blood flow suggest that there is a complex compendium of factors causing the haemodynamic alterations seen in diabetes and further work is needed to unravel the conundrum.

To conclude, this study has shown that increased plasma glucose concentrations after the consumption of a standardised meal test, in subjects with Type II diabetes, result in a corresponding increase of pulsatile ocular blood flow. This fluctuation in blood supply in the presence of hyperglycaemia could increase vascular endothelium cell damage of the choroid and propagate further degradation. This alteration in supply could be a contributory factor in the development of retinal hypoxia and the progression of diabetic retinopathy.

Acknowledgements. R. L. Perrott is funded by The College of Optometrists.

\section{References}

1. Bursell SE, Clermont AC, Kinsley BT, Simonson DC, Aiello LM, Wolpert HA (1996) Retinal blood flow changes in patients with insulin dependent diabetes mellitus and no diabetic retinopathy. Invest Ophthalmol Vis Sci 37: 886-897

2. Langhans M, Michelson G, Groh MJM (1997) Effect of breathing $100 \%$ oxygen on retinal and optic nerve head capillary blood flow in smokers and non-smokers. Br J Ophthalmol 81: 365-369

3. Grunwald JE, Riva CE, Brucker AJ, Sincalir SH, Peitrig BL (1984) Altered retinal vascular responses to $100 \%$ oxygen breathing in diabetes mellitus. Ophthalmology 91: 1447-1453

4. Mendivil A, Cuartero V, Mendivil MP (1995) Ocular blood flow velocities in patients with proliferative diabetic retinopathy and healthy volunteers: a prospective study. Br J Ophthalmol 79: 413-416

5. Grunwald JE, Riva CE, Sinclair SH, Brucker AJ, Petrig BL (1986) Laser doppler velocimetry study of retinal circulation in diabetes mellitus. Arch Ophthalmol 104: 991-996

6. Kunno S, Feke GT, Yoshida A, Fujio N, Goger DG, Bunzey SM (1996) Retinal blood flow changes in type 1 diabetes - a long term follow up study. Invest Ophthalmol Vis Sci 37: 1140-1148

7. Grunwald JE, Riva CE, Martin DB, Quint AR, Epstien PA (1987) Effect of insulin induced decrease in blood glucose on the human diabetic retinal circulation. Ophthalmology 94: 1614-1620

8. Grunwald JE, Dupont J, Riva CE (1996) Retinal haemodynamics in patients with early diabetes mellitus. Br J Ophthalmol 80: 327-331 
9. Yoshida K, Feke GT, Morales-Stoppello J, Collas GD, Goger DG, Mc Meel JW (1983) Retinal blood flow alterations during progression of diabetic retinopathy. Arch Ophthalmol 101: 225-227

10. Schmetterer L, Wolzt M (1999) Ocular blood flow and associated functional deviations in diabetic retinopathy. Diabetologia 42: 387-405

11. Feke GT, Tagawa H, Yoshida A et al. (1985) Retinal circulatory changes related to retinopathy progression in insulin dependant diabetes mellitus. Ophthalmology 92: $1517-1522$

12. DCCT (1993) The effect of intensive treatment of diabetes on the development and progression of long term complications in insulin dependent diabetes mellitus. $\mathrm{N}$ Engl $\mathrm{J}$ Med 329: 977-986

13. Larsson LI, Alm A, Bergenheim T, Lither F, Bergstram R (1999) Retinopathy in diabetic patients aged 15-50years in the country of Umgea Sweden. Acta Ophthalmol Scand 77: $430-436$

14. Moyneaux LM, Constantine MI, Gill M, Zikens R, Yue DK (1998) Better glycaemic control and risk reduction of diabetic complications in Type II diabetes: comparison with DCCT. Diabetes Res Clin Pract 42: 77-83

15. Guillausseau PJ, Massin P, Charles MA, Allaguy H, Guventi Z (1998) Glycaemic control and development of retinopathy in Type II diabetes mellitus - A Longitudinal Study. Diabet Med 15: 151-155

16. Hill DW (1989) Ocular and retinal blood flow. Acta Ophthalmol Scand 67: 15-18

17. Langham ME, Farrell RA, O'Brien V, Silver DM, Schilder P (1989) Blood flow in the Human Eye. Acta Ophthalmol Scand 67: 9-13

18. Langham ME, To'mey KF (1978) A clinical procedure for the measurement of the ocular pulse pressure relationship and the ophthalmic arterial pressure. Exp Eye Res 27: $17-25$

19. Silver DM, Farrell RA, Langham ME, O'Brien V, Schilder P (1989) Estimation of pulsation ocular blood flow from intraocular pressure. Acta Ophthalmol Scand 67: 25-29
20. Mackinnon JR, O'brien C, Swa K, Aspinall P, Butt Z, Cameron D (1997) Pulsatile ocular blood flow in untreated diabetic retinopathy. Acta Ophthalmol Scand 75: 661-664

21. Neudorfer M, Goldstein M, Snir T et al. (1998) Choroidal blood flow in diabetic retinopathy. Invest Ophthalmol Vis Sci 39: 4622

22. Becker P, Schmidt KG, Rickmann A, Hammes HP, Kemkes-Mathes B, Pilliunat LE (1997) Ocular pulse amplitude in diabetic patients. Invest Ophthalmol Vis Sci 38: 5773

23. Dallinger S, Findl O, Rami B et al. (1998) Ocular blood flow and glucose plasma concentrations in patients with IDDM. Diabetelogia 41 [Suppl 1]: 1093

24. O'Conner Davies PH (1994) Local Anesthetics. In: Hopkins G, Pearson R (eds) Ophthalmic drugs: diagnostic and theraputic uses. 4th edn. Butterworth and Heinemann, Oxford, pp 124-131

25. Trinder P (1969) Determination of glucose in blood using glucose oxidase with an alternative oxygen receptor. Ann Clin Biochem 6: 24-27

26. North RV, Cooney O, Chambers D, Dolben J, Owens DR (1997) Does hyperglycemia have an influence upon colour vision of patients with diabetes mellitus? Ophthal Physiol Opt 17: 95-101

27. Porta M (1996) Endothelium: The main actor in the remodelling of the retinal microvasculature in diabetes. Diabetologia 39: 739-744

28. Montenegro MH (1998) Retinal blood flow in Diabetes. Int Ophtalmol Clin 1998: 103-110

29. Tooke JE (1996) Endothelium: The main actor or choreographer in the remodelling of the retinal microvasculature in diabetes. Diabetologia 39: 745-746

30. Keen H, Chloverakis C (1965) Metabolic factors in diabetic Retinopathy. In: Graymore CN (ed) Biochemistry of the retina. Academic press, London pp 123-138

31. Atherton A, Hill DW, Keen H, Young S, Edwards EJ (1980)The effect of acute hyperglycaemia on the retinal circulation of the normal cat. Diabetologia 18: 233-237 